\title{
Toward 5D Imaging in an In-Situ Environmental TEM
}

Huolin L. Xin ${ }^{1}$, Lili Han ${ }^{1}$, and Ruoqian $\operatorname{Lin}^{1}$

1. Center for Functional Nanomaterials, Brookhaven National Laboratory, Upton, NY 11973

In situ environmental TEM (ETEM) is a rapidly evolving area and has experienced impressive developments in imaging naomaterials' transformation in response to the change of environments over the past years due to the availability of dedicated environmental TEMs [1] and holder based systems [2]. For example, with help of MEMS-based local heating holders, it is now possible to monitor the subatomic scale changes of nanocatalysts in gases at elevated temperatures [3]. With the tremendous progress made on the sample environment side, the throughput of analytical STEM imaging and spatially resolved EELS spectroscopic imaging becomes a major bottleneck for various applications where real-time 2D and 3D compositional and bonding information is highly needed. Here, in this talk, we present the development of in situ 4D STEM-EELS tomography and its application in the first series of experiments that attempt to unravel the time-dependent 3-D chemical restructuring process of bimetallic nanocatalysts upon oxidation.

Fig. 1 shows the in situ STEM-EELS imaging of a single Fe-Co nanocatalyst upon heating at 500 degree $\mathrm{C}$ in 0.2 mbar of oxygen performed in an environmental Titan. The elemental maps visualize in projection the segregation and oxidation of Fe and Co. Both the maps and the analysis of the Fe/Co L2,3 near edge fine structures suggest that Fe segregates out and gets oxidized prior to that of Co. This is in agreement with the fact that $\mathrm{Co}$ is more noble than $\mathrm{Fe}$ (the standard reduction potential of Co is lower than that of $\mathrm{Fe}$ ). In spite of that, the reaction pathway regarding how cobalt penetrated the Fe oxide shell remain elusive due to the limited 2D projection information. To reconstruct the 3D chemical pathway, we performed in situ STEM-EELS tomography at a series of important reaction stop points using a custom-made MEMS-based high-tilt tomography heating holder (DenSolution inc., Netherlands). This constitutes a discrete version of 5D imaging. In the following, we will describe our development of quantitative STEM-EELS tomography.

Compared with STEM-EDX, the advantage of STEM-EELS is its capacity to extract spatially resolved bonding information. Figure 2 demonstrates this capability using an example of a partially oxidized FeCo catalyst. By applying statistical classification to the Fe L2,3 near edge fine structures, the metallic and oxidized iron component maps can be readily extracted (Fig. 2). In addition, one aspect that EELS traditionally falls short of is that EELS has difficulty working with thick samples due to multiple scattering. We used a quantitative dual-EELS method developed in refs. [4] to correct for both multiple inelastic and elastic scattering artifacts in the tilt series. The fulfilment of the projection requirement not only improves the reconstruction resolution but also allows tomograms to be rendered quantitatively. Fig. 3 gives a reconstruction example of the 3D mosaic structure of the Fe-Co bimetallic nanocatalysts at the end point of its chemical restructuring. [5]

\section{References:}

[1] R. Sharma and K. Weiss, Microscopy Research and Technique 42 (4), 270 (1998).

[2] HL Xin et al, Microscopy and Microanalysis 19 (06), 1558 (2013).

[3] HL Xin et al, Nano Letters 14 (6), 3203 (2014).

[4] HL Xin et al, Utramicroscopy, 19, 38 (2014) and HL Xin et al, Phys. Rev. B., 90, 214305 (2014) 
[5] Research carried out at the CFN/BNL, which is supported by the U.S. Department of Energy, Office of Basic Energy Sciences, under Contract No. DE-SC0012704.
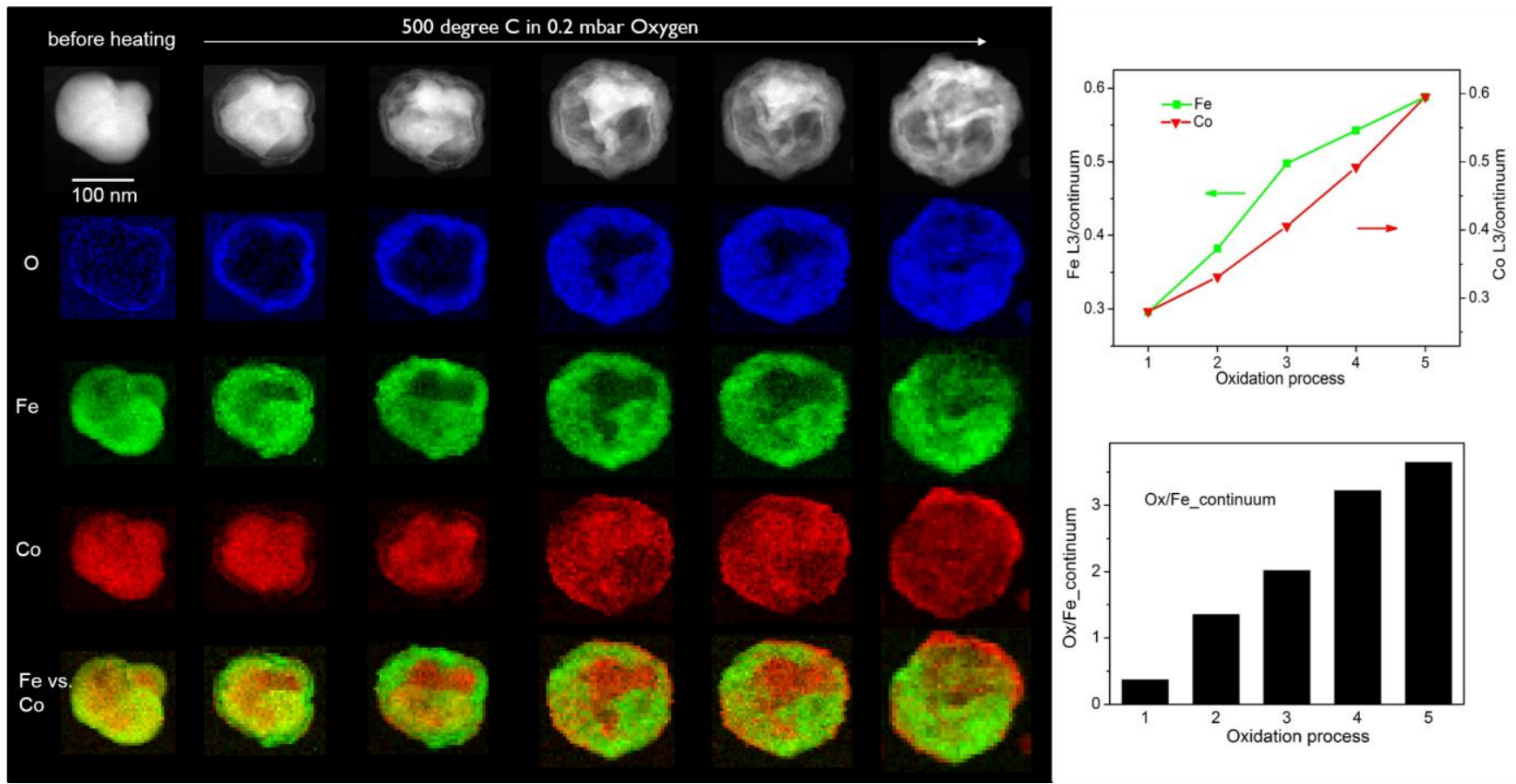

Figure 1. Time-lapsed in situ STEM-EELS imaging of a single Fe-Co nanocatalyts using MEMS-based tomography heating holder.
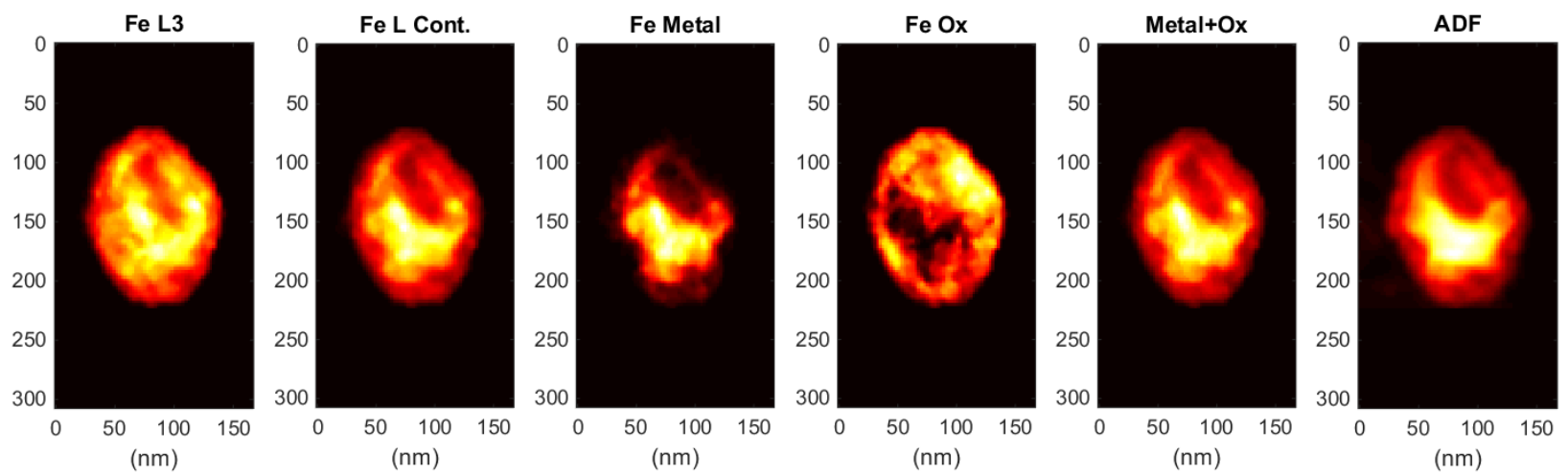

Figure 2. Extraction of Fe metallic and oxidized component maps using linear decomposition of the $\mathrm{Fe}$ L2,3 edge. Results acquired at -30.9 degree tilt for a partially oxidized Fe-Co catalysts (not the same catalyst in Figure 1).

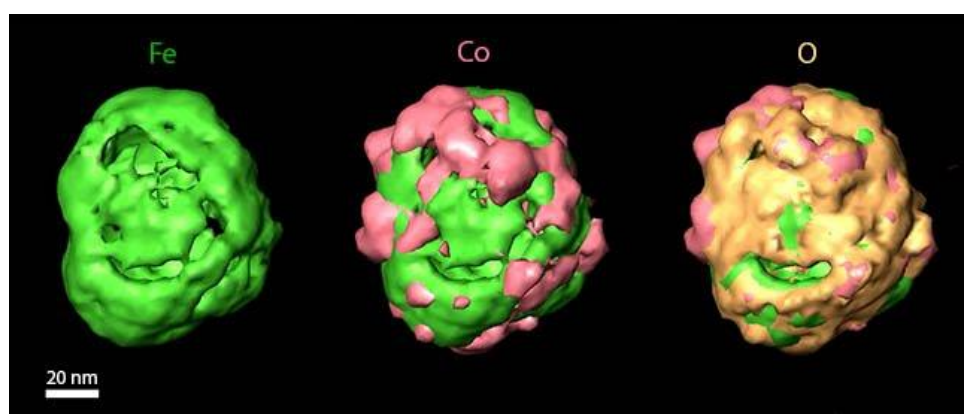

Figure 3. The STEM-EELS extracted elemental reconstruction of a fully oxidized Fe-Co nanocatalysts. 